DOI:10.5216/racs.v5.59357

\title{
Educación matemática y enseñanza para la comprensión: el caso de la cultura hñahñu
}

Vicente Arroyo Aguazul ${ }^{1}$

\section{RESUMEN}

El propósito de este artículo es mostrar que la cultura hñahñu ${ }^{2}$ como otras culturas, poseen características, sociales, lingüísticas, culturales y sobre todo conocimientos propios que los caracteriza y estos conocimientos son poco retomados por la escuela bilingüe indígena, como lo es el caso de la etnomatemática. Los niños hñahñus construyen sus conocimientos tanto en el contexto de las actividades en las productivas en las que él participa y observa en la vida cotidiana así como en los tiempos y espacios escolares. Uno de los problemas que subsiste en la escuela bilingüe indígena y concretamente en la enseñanza de las matemáticas, es la pretensión de desarrollar en los niños y niñas, habilidades y capacidades de manera mecánica y memorística, y no buscan que se vuelvan usuarios y eficientes de las mismas desarrollando el pensamiento matemático a partir de la etnomatemática hñahñu que está presente en las actividades productivas y en la vida cotidiana. Mi perspectiva personal es la posibilidad de incidir y replantear el trabajo académico de la escuela bilingüe indígena en el contexto de la diversidad cultural, en el que los docentes vean como una alternativa, ofrecer una enseñanza que parta de los conocimientos comunitarios como el caso de la etnomatemática que son saberes que pueden apoyar a los alumnos a acceder a las matemáticas escolares.

PALABRAS ClAVES: Etnomatemáticas. Educación bilingüe indígena. Cultura hñahñu.

\section{Educação matemática e ensino para a compreensão: o caso da cultura hñahñu}

\section{RESUMO}

O objetivo deste artigo é mostrar que a cultura Hñahñu, como outras culturas, possui características sociais, linguísticas, culturais e, acima de tudo, possui um conhecimento próprio que a caracteriza. Este conhecimento não é retomado pela escola indígena bilíngue, como se dá no caso da etnomatemática. As crianças Hñahus constroem seus conhecimentos tanto no contexto

\footnotetext{
1 Asesor Pedagógico en la Supervisión Escolar 045, Sector 07 de Primarias Generales. Hñahñu y hablante de la lengua. Región Valle de Mezquital, Estado de Hidalgo, México. E-mail: vice28-12@ hotmail.com.

${ }^{2}$ Cultura Indígena de México, asentado en la región Valle del Mezquital, Hidalgo, México.
} 
das atividades produtivas nas quais participam e observam, no cotidiano, como também nos tempos e espaços escolares. Um dos problemas que permanecem na escola indígena bilíngue, e especificamente, no ensino de matemática, é a reivindicação de desenvolver em crianças, habilidades de uma forma mecânica e apenas para ser memorizado. Não se busca transmitir o pensamento matemático a partir do etnomatemático hñahñu que está presente nas atividades produtivas e na vida cotidiana. Minha perspectiva pessoal é a possibilidade de influenciar e repensar o trabalho acadêmico da escola bilíngue indígena no contexto da diversidade cultural, na qual os professores vêem como alternativa, oferecer educação baseada no conhecimento da comunidade, como o caso da etnomatemática, que são conhecimentos que podem ajudar os alunos a acessar a matemática escolar. Palavras-chave: etnomatemática, educação bilíngue indígena, cultura hñahñu.

Palavras-chave: Etnomatemáticas. Educação bilingue indígena. Cultura hñahñu.

\section{Introducción}

Las matemáticas son parte fundamental de toda cultura y no sólo una colección de herramientas; se deben enseñar como un lenguaje que permita articular las demás ciencias, porque "Las matemáticas son hoy tanto una ciencia como una habilidad necesaria para la supervivencia en una sociedad compleja e industrializada" (CARRAHER, 1997: 48); como una forma de leer, entender y explicar el mundo en el que vivimos. La etnomatemática es entendida como el conjunto de conocimientos matemáticos propios de una cultura, utilizados en la resolución de problemas, según, D’ Ambrosio $(1990)^{3}$ citado por Isaías Aldaz, la etnomatemática son "el arte o la técnica de entendimiento, explicación, conocimiento, abordaje y dominio del medio ambiente natural, social y político que depende de los procesos de contar, medir, clasificar, ordenar, inferir, que resultan de grupos culturales bien identificados”.

Actualmente, la mayoría de las comunidades indígenas hñahñus conservan gran parte de la cultura que heredaron sus antepasados como la elaboración de artesanías y en ellas subyacen conocimientos matemáticos tales como: los morrales de lana, los sombreros de palma, las canastas de carrizo, cobijas de lana, el ayate, etc., aún sabiendo ellos que en el mercado los productos artesanales pierden su valor, sustituidos por otros con la misma función y de menos costo; mucha gente lo sigue trabajando y los vende o hacen el trueque entre sus vecinos. En cuanto a las costumbres: la mayordomía, las fiestas patronales, la fiesta de todos los santos, la entrega de la flor, la forma de curar algunas enfermedades entre otras, se siguen practicando, 
aunque bien se sabe que no son autóctonas, pero fueron practicados por los antiguos hñahñus, también se conservan algunos cantos, leyendas y música del mismo grupo étnico.

Una meta que la escuela bilingüe en el medio indígena debe fijar, es buscar alternativas para vincular los saberes de la población indígena con los conocimientos universales, como una tarea indispensable para lograr que los niños y niñas que asisten a la escuela se apropien de aprendizajes significativos. La educación en el medio indígena desde la perspectiva intercultural, debe apuntar a considerar el proceso educativo como un espacio de reflexión, revaloración, preservación y difusión de su concepción del mundo y de las cosas; donde los grupos indígenas sean considerados como sujetos de su propio desarrollo y no como objetos a formar. En este sentido la educación en el contexto de la diversidad cultural, debe apuntar hacia la revalorización de "lo propio", del conjunto de prácticas y conocimientos que le dan especificidad a un estilo de vida con sus propias formas de producción y reproducción de conocimientos y de prácticas sociales específicas, sin perder de vista la perspectiva del contexto regional y nacional.

\section{El Valle del Mezquital y su contexto geográfico}

El Estado de Hidalgo, según datos de la Secretaría de Programación y Presupuesto, por su tamaño ocupa el lugar número 26 entre las 32 entidades federativas que constituyen los Estados Unidos Mexicanos. Está situado en la meseta central, sus coordenadas extremas son $19^{\circ} 54^{\prime}$ y $21^{\circ} 24^{\prime}$ de latitud norte y los meridianos $97^{\circ} 58^{\prime}$ y $99^{\circ} 54^{\prime}$ de longitud oeste (Ver anexo no. 4). Su longitud máxima, de norte a sur, es de $164 \mathrm{Km}$. su mayor anchura, de 203 y su perímetro total de 1,040. Colinda al norte, con San Luis Potosí; al noroeste, con Veracruz; al este, con Veracruz y Puebla; al suroeste, con Tlaxcala; al sur y suroeste, con México y al oeste con Querétaro, (HIDALGO, MONOGRAFÍA ESTATAL:1996: 14).

El Estado de Hidalgo está conformado por 84 municipios de los cuales 29 conforman la Región del Mezquital, también cuenta con diez regiones geográfico-culturales: Huasteca, Sierra Alta, Sierra Baja, Sierra Gorda, Sierra de Tenango de Doria, Valle de Tulancingo, Comarca Minera, Altiplanicie Pulquera, Cuenca de México y Valle del Mezquital ${ }^{4}$ y tres grupos indígenas: Nahuatl, Tepehua y Hñahñu.

\footnotetext{
${ }^{3}$ D'AMBROSIO, Profesor brasileño a quien se le acredita la acuñación del término ETNOMATEMÁTICA, él ha enfatizado la influencia de los factores socioculturales sobre la enseñanza y aprendizaje de las matemáticas.

${ }^{4}$ Así se denomina la Región por ser un lugar donde abundan los mezquites que son plantas que tienen hojas pequeñas y espinas.
} 
El Valle del Mezquital es una de las diez regiones del Estado con una superficie de 822,000 hectáreas, o sea el 39.4\% del total de la entidad al que pertenece. Está ubicado dentro del Estado de Hidalgo. Según otras fuentes este Valle es una prolongación del valle de México, forma parte de la provincia fisiográfica de la llamada Meseta Neovolcánica y se halla en la zona adyacente a la vertiente occidental de la sierra madre (Cfr. VAZQUEZ, 1995:198). Comprende 29 municipios, de los cuales 15 se encuentran en la zona desértica: Actopan, Alfajayucan, Ixmiquilpan, Nicolás Flores, Nopala, El Salvador, Santiago de Anaya, Tecozautla, Tasquillo, El Arenal, Cardonal, Chapantongo, Chilcuautla, Huichapan y Zimapán; y 14 al área regada: Ajacuba, Atitalaquia, Atotonilco, Mixquiahuala de Juárez, Tezontepec de Aldama, Tepeji de Ocampo, Tetepango, Tlaxcoapan, Tula de Allende, San Agustín Tlaxiaca, Tepetitlán, Francisco I. Madero, Tlahuiltepa y Progreso. El Mezquital está situado básicamente en cuenca del Río Tula, que es la corriente de agua principal, en la vertiente oriental el Río Moctezuma es marginal y corre casi fuera de los confines del Valle del Mezquital.

El fondo del Valle, tiene un subsuelo profundo que ha sostenido la agricultura desde tiempos prehispánicos, por lo que la vegetación está muy alterada; predominan actualmente aparte de los campos cultivados, arbustos espinosos como el nopal, el garambullo, la biznaga, el cardón, el órgano, la palma y otros arbustos fibrosos como la lechuguilla y el maguey; entre los árboles no espinosos está el pirul, el mezquite con algo de espinas en el tallo. La planta más generosa es el maguey ${ }^{5}$, útil anteriormente en la construcción de casas y para el fuego; el aguamiel, donde se obtiene el pulque; para la fibra que sirve para la fabricación de ayates.

Las pendientes de los montes presentan una flora que varía no solamente en razón de la altitud, sino también, respecto a los vientos y a la composición geológica. Por las características geográficas de la región, su fauna se ve disminuida, solamente cuenta con algunos animales domésticos como el perro, el gato, el cerdo, el chivo, gallinas, el burro, el borrego, la res, el guajolote y los animales del campo como el coyote, el conejo, la liebre y algunos roedores. La topografía dominante de la región es planicie y algunos que otros cerros que se observan en los alrededores del valle. El clima que predomina, es templado, la temperatura promedio es de $16.3^{\circ} \mathrm{C}$. La precipitación pluvial anual es de $45 \mathrm{~mm}$ aproximadamente.

\footnotetext{
${ }^{5}$ El maguey, el nopal, el órgano y la lechuguilla son cactus propios de la Región Valle del Mezquital, que es una zona árida con clima cálido seco.
} 


\section{Los hñahñus: su historia cultural y social}

Hay que destacar que el nombre que el grupo Náhuatl le asignó a este grupo étnico desde un principio, es otomitl u otomite, que significa hombre valiente, grosero y peleonero. En la actualidad, los etnolingüístas y otros conocedores de la cultura se han dado a la tarea de indagar el verdadero nombre de esta etnia y han llegado a la conclusión que el grupo se llama a sí mismo "HÑAHÑU", que significa HÑA -habla- y HÑU -nariz- (fosas nasales); el que habla utilizando las fosas nasales.

Antes de la llegada de los españoles el grupo hñahñu ya estaba en el Valle de México, fue uno de los primeros grupos que llegaron a Mesoamérica y se dice que vinieron del norte de México. Este grupo indígena no era guerrero, sino al contrario, muy pacífico. Ellos empezaron a practicar la agricultura desde tiempos muy remotos y también tenían una cultura muy desarrollada, además les gustaba la música, la cual los caracterizaba.

Después llegaron otros grupos procedentes también del norte del país, entre ellos los nahuas, purépechas, etc. Cuando llegaron los nahuas, ya los hñahñus estaban ocupando el Valle de México. Como los nahuas, los cuales sí eran guerreros, conquistaron a este grupo y lo tuvieron bajo su dominio. Algunos de ellos al estar bajo el dominio de los nahuas, huyeron a lugares a donde no los sojuzgaran, fue como se establecieron en diversos lugares. Por ello, actualmente el grupo hñahñu se encuentra disperso en diferentes Estados de la República Mexicana: Querétaro, Guanajuato, Estado de México, Hidalgo, Puebla, San Luis Potosí, Tlaxcala y Veracruz.

\section{Indumentaria}

El traje tradicional para el hombre hñahñu, consta de camisa y pantalón blanco, elaborado por las mujeres de la familia o por costureras locales. La tela es de manta y como calzado se usa el guarache. La mujer viste falda y blusa con vistosos bordados. En ocasiones, para las fiestas, usa el quechquémitl hñahñu. Esto se complementa con el ayate que se pone en la cabeza, que es la prenda de vestir más típica de la región. Ahora la indumentaria tradicional es desplazada por la ropa de moda en los gustos de la nueva generación, las pocas personas que la usan, generalmente son de la generación adulta. 


\section{Vivienda}

Anteriormente, la vivienda se construía con los recursos que proporcionaba el medio ambiente, como son el barro, el órgano, pencas de maguey, palma, entre otros. Por lo regular las casas eran de forma rectangular, sin ventanas, con techo de dos aguas, una puerta de entrada muy baja, con aplanado de tierra en algunos casos y otros materiales que se obtenían en el medio. Actualmente se observa la sustitución de los elementos locales por los comerciales: cemento, ladrillo, lámina de cartón, asbesto o metálica. En estos últimos años se ha incrementado estos cambios por las posibilidades económicas de la gente que ha emigrado a Estados Unidos de Norteamérica, por ende la actividad de la albañilería ha sido una buena fuente de empleo para los que practican la albañilería.

\section{La lengua hñahñu presente entre sus hablantes}

En la actualidad, la mayoría de las familias aún se comunican en lengua hñahñu, lo mismo se hace en una convivencia familiar, encuentro, saludos, faena o reunión que se hace en la comunidad.

La población de las comunidades del Valle del Mezquital son en su mayoría bilingües, el uso del español es considerado como medio para hablar con las personas que son de fuera o que demuestran no hablar o dominar el hñahñu. El desplazamiento de la lengua hñahñu ha sido más notorio en estas últimas décadas, dada la influencia que reciben de los medios de comunicación como la televisión y la radio. Algunos han optado por usar más el español, pensando en que la cultura y la lengua indígena es inferior a la cultura y lengua oficial. Hay casos de personas que emiten un rechazo hacia la cultura y la lengua a la que pertenecen, también tratan que sus hijos dominen la lengua nacional, que se olviden de la lengua materna, porque, según ellos el que habla hñahñu no es aceptado por la sociedad dominante. Algunos sí saben dominar esta situación y aceptan las dos lenguas.

La escuela contribuye que la lengua no se use en los diferentes espacios donde interactúan los hablantes de la lengua hñahñu, porque algunos maestros le restan importancia al bilingüismo, al contrario, evitan que en el aula se hable la lengua indígena; así es como empieza el alumno a privilegiar la lengua nacional y a rechazar la lengua indígena. Se ha observado inclusive que algunos maestros niegan su identidad y no nada más eso, sino que influyen en sus alumnos y en sus hijos para que dejen de usar la lengua que identifica al grupo.

R. Articul.const.saber, 2020,v.5: e59357 


\section{Formas de organización y vida comunitaria}

La autoridad en las localidades del Valle del Mezquital, está constituida jerárquicamente por un representante que es el Delegado Municipal y algunos comités que llegan a constituirse, uno de ellos es el de la escuela para la dirección de algunas obras y demás trabajos, también hay un comité de agua potable. El Delegado Municipal, programa y aprueba coordinadamente con la comunidad algunas acciones, como: Faenas 6 , aportaciones económicas, acuerdos. Para la elección de un ciudadano a estos cargos, el pueblo se reúne con previo aviso, para el nombramiento de las autoridades: la delegación municipal, el comité de agua potable, un comité por cada centro educativo, un comité de salud, entre otros. El Delegado Municipal, tiene un período de un año, lo mismo con sus componentes: el delegado suplente, el juez varista primero, segundo y tercero, pero también varía la cantidad de elementos y los cargos de un pueblo a otro.

\section{La economía de las comunidades hñahñus}

En cuanto a la tenencia de la tierra, prevalece la pequeña propiedad en la mayoría de las comunidades; el ejido y la propiedad comunal en algunas. En esta última, no se produce nada, ya que son terrenos accidentados y laderas; solamente se saca piedra para la construcción de casas y otra parte sirve de pastizales.

La agricultura de esta región, en su mayoría, es de temporal, por lo que se obtiene una cosecha por año. Los principales cultivos, son: el maíz criollo, que se siembra junto con el frijol y el haba. En algunas comunidades se explota el maguey y para muchos constituye la base económica, lo hacen vendiendo el pulque, éste se obtiene a partir de todo un proceso: el "tlachiquero" o el "raspador", es la persona que se encarga de ir todos los días por la mañana, mediodía y tarde a sacar aguamiel del maguey, para depositarlo en un lugar específico y ahí mismo se fermenta, convirtiéndose en pulque. El nopal es también un medio de vida para la gente, ya que de éste se aprovecha la tuna ${ }^{7}$ en su temporada que se saca al mercado para venderla y para el consumo familiar. La lechuguilla, es otra planta que se le saca la fibra a sus penquitas

\footnotetext{
${ }^{6}$ La faena es un sistema de trabajo colectivo para realizar obras que beneficia a la comunidad, donde todos tienen la obligación de participar un día a la semana o cada vez que se requiera. En algunos lugares como Oaxaca, también lo practican y lo conocen como Tequio.

${ }^{7}$ Es el fruto del nopal que es comestible después de quitarle las espinas y la cáscara.
} 
para venderlo o hacer mecapales y lazos. También existe todo un proceso para la explotación de esta planta y elaboración de los productos.

La cría de animales domésticos, es para consumo familiar y para comerlos en días de fiestas preparando mole y barbacoa que son las comidas típicas de la Región. Se puede afirmar que la mayoría de las familias se dedican a esta actividad en sus hogares y en los lugares donde hay suficiente campo se practica el pastoreo como una actividad productiva.

Otras de las ocupaciones de la población, son: jornalero y tlachiquero ${ }^{8}$, se practican generalmente juntas. Sin embargo, estas actividades no son suficientes para obtener todos los productos de consumo familiar, por lo tanto tienen que complementarlas con trabajos asalariados de peones u otras actividades. Mucha gente, al no encontrar trabajo en su propia comunidad, tiene que emigrar a la ciudad en busca de empleo. Si se dan cuenta que ahí perciben bajo salario, dejan el trabajo y entonces emigran a la nación del norte en busca de una fuente de empleo con "buena remuneración", en donde permanecen un buen tiempo, todo por garantizar su porvenir y la de sus hijos.

Las actividades de la mujer son: ocuparse del hogar, del pastoreo y del cuidado de sus hijos. Aunque actualmente se ha observado que tanto el hombre como la mujer emigran a Estados Unidos en busca de un empleo, con el argumento que "lo que percibe el señor de la casa ya no les alcanza para los gastos del hogar".

En todos los tiangüis o plazas del Valle del Mezquital, existe la comercialización desventajosa, donde los pequeños productores se concentran, llevando productos que son arrebatados y pagados con bajo costo por los comerciantes acaparadores. De una manera u otra, la mayoría de los productos son comprados al mínimo precio. Así viven los intermediarios y grandes comerciantes, engañando al indígena, aprovechando estos días de tiangüis que se realizan periódicamente en determinados lugares en el seno del Valle del Mezquital. Lo que más se observa en los tiangüis, es la desvalorización de los productos agrícolas, ganaderas y artesanales que fabrica la gente de la región.

\footnotetext{
${ }^{8}$ Es la persona que extrae la miel del maguey, éste deja un tiempo para que se fermente, se convierte en una bebida que se llama pulque ya con un cierto grado de alcohol que emborracha si se toma en exceso.
} 


\section{La socialización en la familia hñahñu, identidad y conocimientos}

La familia es una unidad económica y de convivencia bien definida, basada en el respeto a los padres. En ella se conservan los valores culturales del grupo étnico, como el respeto que hay en los jóvenes hacia sus mayores y viceversa, además de la presencia y práctica de las costumbres y tradiciones. La familia sigue siendo la célula de un pueblo, subsiste el término en hñahñu, "n'a ra m'ui" que significa una vivienda, donde hay un grupo de parientes asentados en un lugar definido. Es en este contexto donde existe una conservación de la identidad hñahñu.

En esta región Valle del mezquital, por ser una zona árida, hay pocas posibilidades de producción de alimentos, pero el hñahñu ha sabido aprovechar algunos recursos de apoyo para el tratamiento de la tierra y elaboración de artesanías u otros trabajos que se describen en este trabajo.

En la socialización familiar, los niños empiezan a recibir una educación que tiene que ver con los valores, las costumbres y los procesos de cada trabajo, porque también participan en las actividades diversas en el campo y en la casa misma, en algunos casos también con trabajos remunerados como la albañilería o en los trabajos agrícolas.

El jefe de la familia que es el padre de la casa, generalmente es el encargado de emigrar a alguna ciudad cercana, a otro estado o a Estados Unidos, en busca de trabajo para llevarle a su familia alimentación con la venta de su fuerza de trabajo donde le pagan de entre 2 a 4 salarios mínimos, aún cuando tiene que alimentar de 4 a 6 hijos comprando algunos alimentos básicos como son: el maíz, el frijol, la sal, entre otros. Lo cierto es que con esto, el jefe de familia, no puede solventar otros gastos secundarios: vestido, calzado, la educación, etc. De ahí que las familias hñahñus, son víctimas de la pobreza extrema, del desempleo, la marginación y otras políticas económicas, sociales y educativas.

\section{La etnomatemática en las actividades productivas del pueblo hñahñu}

Todo pueblo originario tiene una manera de hacer matemáticas en los diversos contextos de su entorno cultural. En este sentido, el término etnomatemáticas se viene utilizando con mayor énfasis en las últimas dos décadas, también bajo la idea de que cada grupo cultural independientemente del contexto social en el que vive, desarrolla sus propias matemáticas. En el caso de nuestro continente, en la cultura Maya, la matemática alcanzó un gran desarrollo en comparación con otras culturas de su época. Precisamente el sistema de numeración escrito en 
base veinte, creado por los Mayas, ha sido considerado como uno de los cuatro sistemas originales de numeración escrita de posición existentes en el mundo (el de los Mayas, el de Babilonia, el de China y el de la India). Lo mismo ocurrió con el imperio Incaico, desarrolló una cultura matemática cuyo desarrollo fue bloqueado por la conquista española. Después de cinco siglos de marginación y sometimiento cultural, las manifestaciones de la cultura matemática incaica, son conservadas en parte por las comunidades del Antiplano.

México, se caracteriza como un país pluricultural, los pueblos autóctonos que habitamos en esta nación poseemos una cultura propia y por ende una cultura matemática que se pone en práctica en las actividades cotidianas de manera explícita e implícita. Aunque también se da una sobrevaloración de las matemáticas europeas; ZALAVSKY (1989) reconoce que "Todos nosotros, nutridos desde la tradición de la cultura occidental, tendemos a pensar sobre las matemáticas como un único florecimiento de la cultura europea...Sin embargo, evidencias culturales surgieren que la matemática ha florecido en todo el mundo, y que los niños se benefician de ella aprendiendo como prácticas matemáticas provenientes de las necesidades reales y deseos de las sociedades".

La etnomatemática es un término que también tiene diferentes conceptos, así para (ASCHER, et. al. 1988), la "Etnomatemática es el estudio de las ideas matemáticas de gente no letrada". Para algunos este término tiene un reconocimiento más específico. "Dado que la matemática es una ciencia que pertenece al patrimonio de la cultura universal, cuyos rudimentos se remontan a los orígenes del hombre, y cuyo desarrollo actual se debe al aporte de varias civilizaciones de Oriente y Occidente, la etnomatemática de un grupo social determinado estaría constituida por casos particulares de algunos modelos matemáticos, ligados a su cosmovisión" (VILLAVICENCIO, 1987).

Para poder recuperar estos conocimientos, se requiere hacer investigaciones de manera exhaustiva para establecer las diferencias sobre los usos de la matemáticas en contextos particulares como es el caso de los grupos étnicos en nuestro país. Con esta idea se retoma el siguiente planteamiento: "La precisión conceptual de ETNOMATEMÁTICA se logrará en función de una mayor investigación en este campo. En todo caso, lo positivo a rescatar es la creciente conciencia de la importancia de considerar en la educación formal los conocimientos y técnicas matemáticas del grupo cultural al cual pertenece el educando como medio para mejorar el aprendizaje de la Matemática, superando la alienación cultural” (VILLAVICENCIO, 1990: 91). 
Por ello el sentido de este trabajo, tiene que ver con la importancia de reconocer que en la cultura hñahñu, existen conocimientos etnomatemáticos propios, que son empleados en las actividades cotidianas para resolver problemas que implica medir, contar, agrupar, entre otros, mismos que pueden ser recuperados en la escuela.

En apartados anteriores se ha hecho referencia que todo grupo cultural tiene conocimientos etnomatemáticos como parte de la cultura y cosmovisión que le ayuda a vivir y actuar en el medio donde habita, este es el caso de los hñahñus, en el contexto de la vida cotidiana también tienen una manera de contar, medir longitudes, medir el tiempo y de explicar cosas en términos de números y operaciones. Lo único que ha faltado es escribir y sistematizar ${ }^{9}$ estos conocimientos, dado que se rigen por procesos que implican un orden que si no se respeta, simplemente no se logra el producto esperado. Este es el caso de los conocimientos que se emplean para desarrollar algunas de las actividades productivas que se mencionarán en los siguientes párrafos.

En el contexto de la vida cotidiana de los hñahñus, se realizan un sinnúmero de trabajos, actividades y tareas para la sobrevivencia del mismo, con ellos también hay un manejo de números y operaciones. Los números suelen tener distintos significados dependiendo del contexto particular en donde se emplea. "Diferentes contextos numéricos (contextos particulares en los que se emplean los números), son, según acabamos de ver, secuencia verbal, contar, cardinal, medida, ordinal, como código y, finalmente, producto de la era electrónica, aparece el número como tecla, botón o resorte" (CASTRO, 1989:97). Dichos contextos pueden encontrarse de manera aislada o abarcando más de uno de los significados.

Los pobladores de la región desarrollan varias actividades productivas, aquellas que se realizan como trabajo y obtención de productos ${ }^{10}$ para el ingreso económico familiar y para hacer trueques (intercambio de cosas). Existen procesos que se siguen para la realización de las actividades productivas, se manejan una serie de conocimientos sobre la calidad del material, elaboración y formas de obtener mejores resultados de los productos e implícitamente un lenguaje matemático. Un caso concreto son los conocimientos que hay en torno a la siembra que es una actividad milenaria, mismos que son transmitidos de generación en generación.

\footnotetext{
${ }^{9}$ La sistematización es la organización de los datos que permiten conocer el desarrollo de un acontecimiento.

${ }^{10}$ En la cultura hñahñu, siempre que la gente realiza alguna actividad, espera obtener un producto, para ello realizan las actividades de una manera sistemática. El proceso de sistematización que siguen no necesariamente tiene que estar escrito en algún papel, sino que son conocimientos que forman parte de los saberes comunitarios.
} 
"Para sembrar maíz primero se prepara bien la tierra. La tierra tiene que barbecharse, para que la hierba que se cae con la yunta se seque cuando haya heladas. Cuando llega el mes de siembra se escoge el maíz que va a sembrarse, ...Hay que saber si el maíz que se siembra es de temporal o de riego, porque es mejor sembrar de temporal (que se cosecha más rápido) para que no se pierda con las heladas. Este maíz se siembra en el mes de junio y a los cuatro meses ya se puede cosechar. Para que el maíz crezca y se dé bien tiene que sembrarse en luna llena y no cuando sea luna nueva" (MARTIN, et. al, 1986:16).

Como puede observarse, en este proceso se emplea una serie de conocimientos tales como, el manejo de los tiempos: cuando se habla de temporadas de heladas, meses que son propicios para sembrar; también se manejan cantidades: cuando se habla de meses que se prolongan para la cosecha y por ende la cantidad de terreno y de maíz a emplear.

De igual forma el proceso que se sigue para la elaboración del ayate ${ }^{11}$ se inicia desde el conocimiento del ciclo de vida del maguey, que es una planta que se explota para obtener pulque y se aprovecha la penca para obtener fibra, conocida como santhe, hasta el dominio del proceso para la elaboración del ayate y la preparación del pulque.

"Para la elaboración de un ayate es necesario utilizar el Santhe o Ixtle. Ixtle , se saca tallando las pencas maguey; estas pencas deben ser las corazón del maguey y deben estar cortadas un mes antes, para que se enjuten con tiempo y no se tengan asar. De esta manera el Ixtle queda Cuando se necesite asar las pencas maguey, se coloca el maguey en un que tapan con tierra durante algunos días. Esto se hace para que tallar las pencas se caiga más la corteza del maguey, que se talla

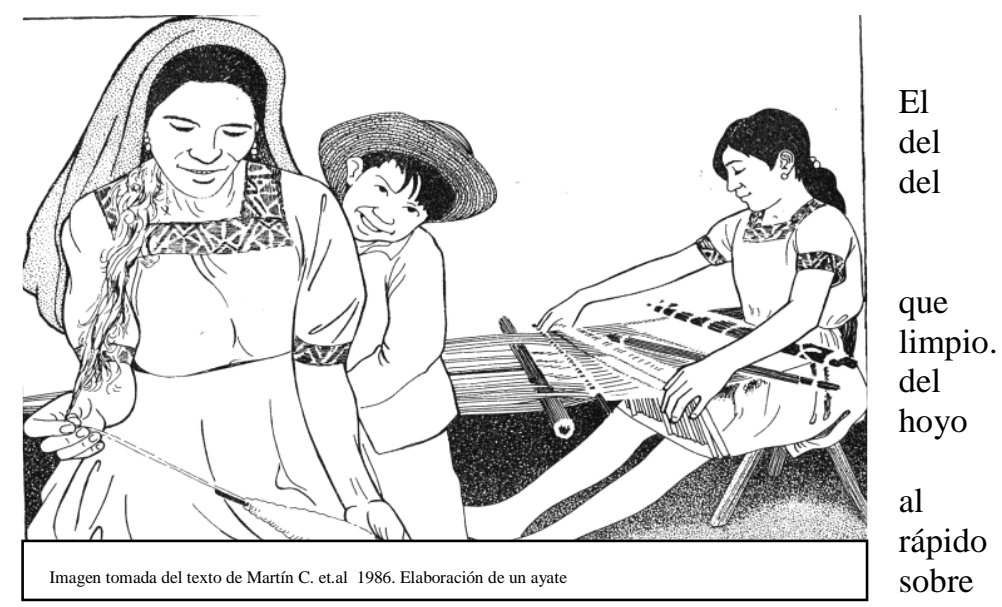
una tabla. Cuando ya está listo el ixtle se lava bien le quita la basura y después se hila. Para poder hilarlo es necesario buscar un pedazo de palo y una piedra redonda con un hoyito donde se pueda meter el palo y así poder construir el malacate. Este sirve para darle vueltas y poder torcer el hilo: si se quiere delgado se hace con dos hilos si se quiere grueso se hace con tres hilos. Cuando se tenga una buena cantidad de hilo torcido se puede empezar a hacer el ayate. Para tejer un ayate se necesita el tejedor. El nopal, el yab'e, el juts'i, el njat'i y el peine. Primero se pone el juts'i con el yab'e, luego ponen el peine con el nopal, también ponen el ixtle en el peine y así se empieza a tejer.Se levanta el juts'i para que tenga el joi y meten el ixtle en el njät'i varias veces para que se junte el ixtle, hasta terminar el ayate. Una vez terminado el ayate es pasado con una púa de maguey para que no queden hilos desiguales; luego lo enrollan y lo estiran de los extremos para que no quede encogido". (MARTIN, et. al, 1986:16).

El fragmento anterior muestra que en la elaboración del ayate, también se requiere saber el nombre de los implementos, los usos de estos, la cantidad del material a emplear, la calidad de 
la misma, las medidas no convencionales ${ }^{12}$ que se usan para hacer un ayate del tamaño deseado, la forma del tejido, las características y tamaño del tejedor que se usa, entre otros.

La barbacoa es otra de las actividades que realizan algunas personas es la preparación de la barbacoa que es una comida típica de la región; en algunos casos resulta ser una fuente más de ingresos económicos dado que algunos se dedican a vender este producto en los mercados o en las orillas de las carreteras donde hay mucha afluencia vehicular. También se observa que la mayoría de los que son "barbacoyeros"13, son contratados o invitados en las fiestas para este trabajo, porque no muchos poseen los conocimientos y la aptitud para esta actividad, debido al esfuerzo, los riesgos y los compromisos que implica su realización.

"Para preparar la barbacoa, hay que saber matar los chivos, los borregos, o la res según
lo que los dueños de la casa quieran preparar; luego hay que preparar un hoyo para echar
la carne y unas cuarenta pencas o más según sea la cantidad de carne, por ejemplo
cuando se echa como unos cinco chivos más o menos medianos se requiere unas
cuarenta pencas de maguey, éstas pencas hay que quemarlas para que se vuelvan frágiles
y también para que le dé un buen sabor a la carne. También hay que calentar bien el
hoyo y las piedras al rojo vivo, echándole mucha leña desde una dos horas antes de
enterrar la carne. Hay que saber a qué horas se quiere la barbacoa por ejemplo si la
barbacoa la quieren para las dos de la tarde hay que enterrarla desde las ocho o nueve de
la mañana, dependiendo también de los animales si eran tiernos o no, pero yo le calculo
como unas cinco horas para que esté lista la barbacoa, porque también se sabe desde
fuera si el horno empieza a sumirse un poco, eso quiere decir que la carne ya se coció,
entonces ya no se le pone más lumbre, ya esta lista la barbacoa para sacarla y
servirla..."'(SIC. SR. ANDRÉS)

Esta es una actividad que además de los conocimientos que se requieren: matar los animales, cortar y quemar las pencas de maguey, preparar el hoyo, quemar las piedras y calentar el hoyo; también se necesita afrontar los riesgos y las responsabilidades tales como soportar el calor de la lumbre, hacer bien los cálculos para que la carne se cosa bien, calcular los condimentos que se le echa al consomé y por último cumplir con el encargo de ofrecer la comida principal para la fiesta que puede ser de tipo religioso, familiar o cualquier evento.

Por lo que se ha observado es un número elevado de tiempos y ocasiones en que los niños emplean para observar y participar en esta actividad, dado que en cada momento se congregan la

\footnotetext{
${ }^{11}$ El bordaje del ayate se convierte en una prenda como especie de pequeño cobertor con medidas aproximadamente de 1:20 x 1:120 cm. Se usan para cargar cosas en la espalda adecuándolo con un mecapal.

12 En la elaboración de un ayate se utilizan "la cuarta" que mide aproximadamente $19 \mathrm{~cm}$. y "el codo" que mide aproximadamente $15 \mathrm{~cm}$.

6 Este es el nombre que se le asigna a aquellos que tienen conocimientos sobre cómo preparar la barbacoa a través de un horno bajo tierra, es una comida típica de la región. Trabajo que inicia desde el sacrificio de los animales, preparación del horno, hasta la obtención de la carne ya cocida en barbacoa.

${ }^{14}$ Señor entrevistado sobre su amplio conocimiento para preparar la barbacoa.
} 
mayoría de los niños de las familias que acuden a ayudar con los preparativos de la fiesta y también es notorio la frecuencia de estos eventos durante el año: bautizos, fiesta familiar, matrimonios, primeras comuniones, quince años, clausuras, entre otras fiestas, por ello los niños adquieren buena experiencia y saberes que la escuela puede recuperar. Lo interesante de todo este proceso es la tarea misma, los conocimientos que se manejan tales como: el tanteo, el volumen, la superficie, el manejo del espacio y del tiempo, el cálculo (sobre los tiempos de preparación y la cantidad de carne que rinde para cierta cantidad de personas de una fiesta), la serie numérica, las medidas no convencionales, entre otros. Varios de estos conocimientos manejados en las actividades productivas, también se trabajan en la escuela primaria como contenidos escolares.

Otras de las actividades productivas que más se practica en la región, es la albañilería, esta actividad se aprende, "primero observando y ayudando a los que ya saben y tienen mucha práctica". Para conocer más sobre esta actividad se entrevistó a Don Carlos, un señor que se hizo albañil después de que salió de la secundaria a los 17 años, ahora él tiene 37 años. Cuando él se le entrevistó preguntándole sobre cómo se aprende el oficio y qué conocimientos básicos el aprendiz puede colaborar con un maestro albañil, al respecto dijo lo siguiente:

Lo primero es tener ganas de trabajar, saber utilizar la pala, agarrar el martillo, conocer el metro y saber colocar un hilo, saber plomear y saber medir con el metro, con la cuarta o con el hilo mismo. Cuando uno se inicia en el oficio, empieza uno como chalán (ayudante de albañil), para ser ayudante hay que saber batir la mezcla, saber cuántos botes de arena se le hecha para un bulto de cemento, cuánto de graba, si es revoltura para colar, o cuánto de calidra si es mezcla para pegar tabique o block, porque el maestro solamente te explica algunas veces, no siempre lo va a repetir. Como maestro de media cuchara, el maestro contratista o patrón te va a dar algunos trabajos más complicados donde tú tienes que levantar un muro, pegar tabique o block, aplanar, ahí sí tienes qué conocer más cosas, por ejemplo, desde darle el presupuesto, de materiales, de mano de obra, ahí depende si trabajas por día o por un tanto de destajo, también debes de saber medir, sacar escuadra, sacar ángulos, plomear, por ejemplo si te piden que inicies una construcción desde el cimiento, tienes que trazar primero el terreno, levantar el mamposteo, armar las zapatas con medidas especiales de las varillas Hay de media pulgada, de $3 / 8$, de pulgada, también los anillos son de diferentes medidas depende de la medida de los castillos, hay de $15 \mathrm{~cm}$. Por $10 \mathrm{~cm}$, de $20 \mathrm{~cm}$. Por $20 \mathrm{~cm}$. de 25 por 20 según lo que te pida las medidas de los castillos, las trabes, las cadenas. Para levantar un muro hay que saber plomear, conocer la medida de los tabiques y blocks, porque hay algunos que miden 19 por $40 \mathrm{cms}$., algunos 20 por $40 \mathrm{cms}$, y saber cuánto de mezcla ponerle entre cada block para que llegue a la altura que se te pide, porque sino puede rebasar o terminar desnivelado las hileras...Para ser maestro oficial o de cuchara, es necesario saber más, porque ahí sí ya te puedes echar el compromiso de cimbrar o colar una losa o forrar un piso, enyesar o poner loseta. Hay que saber sacar metros cuadrados y saber cubicar, saber armar las varillas para un techo, bayonetear porque esto sí siempre va a ser necesario en todos lados...también hay que entenderle un poco al plano porque qué tal si te encuentras con un arquitecto que te da chamba, ni modo de decirle que no 
sabes, cuando sí sabes hacer las cosas, pero esto se aprende viendo o preguntando, por ejemplo yo lo aprendí con los que han sido mis maestros, anduve en México y otros lugares donde yo he estado trabajando. (Sic. Don Carlos).

Como se puede apreciar, en esta actividad están implícitos varios conocimientos y conceptos matemáticos tales como, el área, la suma, el perímetro, el volumen, la suma, la resta, la división, las fracciones, el ángulo, las formas de las figuras geométricas y los prismas, entre otros contenidos. De igual manera se pone en práctica y se desarrollan varias habilidades tales como el cálculo, la estimación, la comparación, la igualación, la seriación, entre otras. Quien practica este oficio, generalmente utiliza la etnomatemática en combinación con las matemáticas universales, por ejemplo, cuando alguien empieza midiendo con la cuarta, con un hilo o con una vara, posteriormente recurre al metro para contrastar las medidas, algunos hacen más uso de las medidas no convencionales porque ya saben cuánto equivale en centímetros o metros. Por ejemplo utilizan la cuarta porque sabe que equivale a 19 o 20 centímetros, dependiendo del tamaño de los dedos de quien hace uso de este recurso.

En esta actividad se involucran los niños y en algunos casos también a las niñas cuando ayudan al papá en sus trabajos o cuando los invitan por ahí en algún colado o simplemente cuando construyen o se realiza una reparación en la casa donde viven. Por ello los niños van conociendo y apropiándose de algunos contenidos que se manejan en esta actividad, por ejemplo, los niños ya saben cuántos botes de arena caben en una carretilla, cuántos requiere para preparar la mezcla o la revoltura, saben que un bulto de cemento pesa 50 kilogramos y la calidra 25 kilogramos, también tienen nociones lo que es una altura, una longitud, también se saben los nombre de las herramientas, entre otras muchas cosas. Por ello, si la escuela retoma estos saberes, para el niño le sería muy significativo porque son conocimientos que ya conoce, mismos que ha observado y participado en estas actividades donde adquieren y hacen uso de estos saberes.

Otra de las actividades productivas que realizan algunas personas, es la quema del carbón, que también, lo hacen para vender este producto en el tianguis y obtener un ingreso para el sustento familiar. A continuación se describe el proceso que se sigue para obtener dicho producto:

Para hacer carbón se cortan palos de mezquite, de b'inza ${ }^{15}$ y a veces de encino y se cortan en pedazos uno por uno, de manera que no queden ni muy grandes ni muy

\footnotetext{
${ }^{15}$ B inza. Es el nombre en hñahñu de una planta que se produce en esta región, se aprovecha su tallo para leña y fogata.
} 
chicos todos los palos deben estar maduros para que dure el fuego cuando se quemen. Para quemarlos primero hacen un horno en el campo y les ponen unos palos delgados dentro a manera de casita. Le dejan en medio unos agujeros para poder atizar el fuego con unos palos delgados después se le ponen los palos gruesos que son los que se van a hacer carbón. Cuando terminan de acomodar los palos tapan el horno con pencas de maguey, nopal con heno y otras hierbas, y lo cubren con tierra dejándole unos agujeritos en las orillas del horno para que entre aire y no se apague el fuego. Así los palos arden poco a poco. Para que se haga carbón lo dejan algunos días después de que ha pasado tiempo se empieza a apagar el fuego y entonces pueden sacar el carbón para venderlo. (Martín et. al. 1986:10).

Al igual que otras actividades que realiza la población, para la obtención del carbón implica todo un proceso y la puesta en acción de algunos conocimientos matemáticos que tiene que ver con el cálculo, la porción, la estimación, la medición; porque desde que se corta la leña se cuida que la medida de los pedazos sean más o menos de 30 a 40 centímetros, la profundidad del horno debe de ser de acuerdo a la cantidad de leña, también se mide el tiempo para su cocimiento que es de un promedio de $48 \mathrm{hrs}$. Los que se hacen cargo de este proceso, le calculan que sea dos días y dos noches., están al pendiente de que el fuego en el interior del horno no se apague.

En líneas anteriores se describieron los procesos de algunas de varias actividades productivas que se practican en la región, así como los contenidos y conocimientos matemáticos que subyacen en ellas, esto con la finalidad de reflexionar acerca de la importancia de reconocer que estos saberes están presentes en las actividades cotidianas de la población, por lo que los niños también participan y van adquiriendo estos conocimientos en el contexto familiar y comunitario. Además de valorar la importancia de que la escuela recupere estos saberes e inicie la enseñanza de las matemáticas formales a partir de estos conocimientos y experiencias que los niños hñahñus ya poseen utilizando las formas no convencionales, haciendo uso de medidas arbitrarias, para que logren un aprendizaje más significativo de los mismos.

\section{Formas de conteo hñahñu como un lenguaje matemático propio}

Otro de los conocimientos matemáticos que posee la cultura hñahñu es la forma de conteo, saberes que se pretenden mostrar en este apartado, quizá para algunos es simplemente un manejo local de los números ó una forma propia de nombrar y contar. Lo cierto es que en este grupo se tiene una forma organizada de nombrar los objetos, la serie numérica, las horas, el tiempo, los números, los ciclos etc.

Inicio presentando la serie numérica en las dos lenguas (Hñahñu y español), con la intención de que sea entendible para aquellos que no hablan la lengua hñahñu.

R. Articul.const.saber, 2020,v.5: e59357 
Cuadro No. 1. La serie numérica y la notación lingüística en hñahñu y en español. (Cfr. BERNAL, 1996:187).

\begin{tabular}{|c|c|c|c|}
\hline $\begin{array}{c}\text { LA SERIE } \\
\text { NUMÉRICA }\end{array}$ & $\begin{array}{c}\text { LOS NUMEROS EN } \\
\text { HÑNHNU }\end{array}$ & $\begin{array}{c}\text { INTERPRETACION } \\
\text { ARITMÉTICA }\end{array}$ & $\begin{array}{c}\text { LOS NUMEROS EN } \\
\text { ESPAÑOL }\end{array}$ \\
\hline 1. & N'a. & 1 & Uno \\
\hline 2. & Yoho. & 2 & Dos \\
\hline 3. & Hñu. & 3 & Tres \\
\hline 4. & Goho & 4 & Cuatro \\
\hline 5. & Küt'a & 5 & Cinco \\
\hline 6. & $\mathrm{R}^{\prime}$ ato & 6 & Seis \\
\hline 7. & Yoto & 7 & Siete \\
\hline 8. & Hña’to & 8 & Ocho \\
\hline 9. & Guto & 9 & Nueve \\
\hline 10. & R'et'a & 10 & Diez \\
\hline 11. & R'et'a ma r'a & $10+1$ & Once \\
\hline 12. & R'et'a ma yoho & $10+2$ & Doce \\
\hline 13. & R'êt'a ma hñu & $10+3$ & Trece \\
\hline 14. & R'et'a ma goho & $10+4$ & Catorce \\
\hline 15. & R'ett'a ma kut'a & $10+5$ & Quince \\
\hline 16. & R'et'a ma r'ato & $10+6$ & Dieciséis \\
\hline 17. & R'ett'a ma yoto & $10+7$ & Diecisiete \\
\hline 18. & R'et'a ma hña'to & $10+8$ & Dieciocho \\
\hline 19. & R'et'a ma guto & $10+9$ & Diecinueve \\
\hline 20. & N'ate & 20 & Veinte \\
\hline 40. & Yo r'ate & $2(20)$ & Cuarenta \\
\hline 50. & $\begin{array}{l}\text { Yo r'ate ma r'et'a } \\
\text { (ndenthebe) }\end{array}$ & $\begin{array}{l}2(20)+10 \\
50\end{array}$ & Cincuenta \\
\hline 60. & Hñu r'ate & $3(20)$ & Sesenta \\
\hline 70. & Hñu r'ate ma r'ett'a & $3(20)+10$ & Setenta \\
\hline 80. & Goho r'ate & $4(20)$ & Ochenta \\
\hline 90. & Goho r'ate ma r'et'a & $4(20)+10$ & Noventa \\
\hline 100. & N'a nthebe & 100 & Cien \\
\hline 200. & Yo nthebe & $2(100)$ & Doscientos \\
\hline 500. & Küt'a nthebe & $5(100)$ & Quinientos \\
\hline 1000. & N'a m'o & 1000 & Mil \\
\hline 5000. & Kutt'a m'o & $5(1000)$ & Cinco mil \\
\hline 10000. & R'et'a m'o & $10(1000)$ & Diez mil \\
\hline 20000. & N'ate m'o & $20(1000)$ & Veinte mil \\
\hline 50000. & Yo r'ate ma r'et'a m'o & $2(20000)+10000$ & Cincuenta mil \\
\hline 100000. & N'a nthebe m'o & $100(1000)$ & Cien mil \\
\hline 500000 & Kutt'a nthebe m'o & $500(1000)$ & Quinientos mil \\
\hline 1000000. & N'a m'o ya m'o & $1000(1000)$ & Un millón \\
\hline
\end{tabular}

Fuente: Propio autor. 
En el cuadro 2, se presenta la secuencia del tiempo, es decir, la manera de nombrar las horas, al igual que el español, se nombra el numeral acompañado del tiempo, especificando si es de mañana, medio día, tarde y media noche.

Cuadro No. 2. Las horas en la lengua hñahñu y español., (Cfr. BERNAL, 1996:

\begin{tabular}{|c|c|c|}
\hline LAS HORAS EN HÑAHÑU & $\begin{array}{c}\text { LAS HORAS CON } \\
\text { NÚMEROS }\end{array}$ & LAS HORAS EN ESPAÑOL \\
\hline Nzunga n'a xudi & 1:00 A.M. & Una de la mañana \\
\hline Nzunga yoho xudi & 2:00 A.M. & Dos de la mañana \\
\hline Nzunga hñu xudi & 3:00 A.M. & Tres de la mañana \\
\hline Nzunga hñu ma de xudi & 3:30 A.M. & Tres y media de la mañana \\
\hline Nzunga goho xudi & 4:00 A.M. & Cuatro de la mañana \\
\hline Nzunga kutt'a xudi & 5:00 A.M. & Cinco de la mañana \\
\hline Nzunga r'ato xudi & 6:00 A.M. & Seis de la mañana \\
\hline Nzunga yoto xudi & 7:00 A.M. & Siete de da la mañana \\
\hline Nzunga hñato xudi & 8:00 A.M. & Ocho de la mañana \\
\hline Nzunga guto xudi & 9:00 A.M. & Nueve de la mañana \\
\hline Nzunga r'ét'a xudi & 10:00 A.M. & Diez de la mañana \\
\hline Nzunga r'et'a ma r'a xudi & 11:00 A.M. & Once de la mañana \\
\hline Nzungna r'et'a ma yoho xudi & \multirow[b]{2}{*}{ 12:00 A.M. } & Doce de la mañana \\
\hline Nde ma pa & & Medio día \\
\hline Nzunga n'a nde & 13:00 P.M. & Una de la tarde \\
\hline Nzunga yoho nde & 14:00 P.M. & Dos de la tarde \\
\hline Nzunga hñu nde & 15:00 P.M. & Tres de la tarde \\
\hline Nzunga goho nde & 16:00 P.M. & Cuatro de la tarde \\
\hline Nzunga kut'a nde & 17:00 P.M. & Cinco de la tarde \\
\hline Nzunga r'ato nde & 18:00 P.M. & Seis de la tarde \\
\hline Nzunga yoto nxu'i & 19:00 P.M. & Siete de la noche \\
\hline Nzunga hñato nxu'i & 20:00 P.M. & Ocho de la noche \\
\hline Nzunga guto nxu'i & 21:00 P.M. & Nueve de la noche \\
\hline Nzunga r'et'a nxu'i & 22:00 P.M. & Diez de la noche \\
\hline Nzunga r'ét'a ma r'a nxu'i & 23:00 P.M. & Once de la noche \\
\hline Nzunga r'et'a ma yoho nxu'i & \multirow[b]{2}{*}{ 24:00 P.M. } & Doce de la noche \\
\hline Ma de ra xu'i & & Media noche \\
\hline
\end{tabular}

Fuente: Propio autor.

En el cuadro 3, se suscriben las unidades de medida "no convencionales" para representar volúmenes. Lenguaje que es utilizado por las personas que tienen un alto dominio del hñahñu (monolingüies en esta lengua). 
Cuadro No. 3. Ya t'eni - Medidas, (Cfr. BERNAL, 1996:84).

\begin{tabular}{|l|l|}
\multicolumn{1}{|c|}{$\begin{array}{l}\text { UNIDADES DE MEDIDAS EN } \\
\text { HNHANU (VOLÚMEN) }\end{array}$} & \multicolumn{1}{c|}{$\begin{array}{c}\text { UNIDADES DE MEDIDAS EN ESPAÑOL } \\
\text { (VOLÚMEN) }\end{array}$} \\
\hline N'a fot'ie. & Un puño \\
\hline N'a juada & Un cuartillo \\
\hline N'a media & Veinticinco cuartillos \\
\hline N'a tercio & Cincuenta cuartillos \\
\hline N'a karga & Cien cuartillos \\
\hline N'a docena & Una docena \\
\hline N'a gruesa & Una gruesa \\
\hline N'a kubo & Veinticinco litros \\
\hline
\end{tabular}

Fuente: Propio autor.

También existe una forma propia de contar el dinero, pero de las monedas que existían mucho antes, ahora este lenguaje se emplea menos debido a que ya no existen estas mismas monedas. En la actualidad se emplea la serie numérica para nombrar cantidades y únicamente se le agrega a la cantidad la palabra "Bexo" que quiere decir "Peso" para referirse la una cantidad de dinero.

Cuadro No. 4. Ya mëde bojä- Las formas de contar dinero.

\begin{tabular}{|l|l|}
\multicolumn{1}{|c|}{ FORMAS DE CONTAR EL } & \multicolumn{1}{c|}{ FORMAS DE CONTAR EL DINERO EN } \\
DINERO EN HÑANANU & Tres centavos \\
\hline Nguatra & Seis centavos \\
\hline N'a meñu & Nueve centavos \\
\hline Meñunguatra & Doce centavos \\
\hline Ndomi & Quince centavos \\
\hline Ndominguatra & Veinticinco centavos \\
\hline Ny'odm'i & Cincuenta centavos \\
\hline Ngundm'i & Setenta y cinco centavos \\
\hline R'ato domi & Un peso \\
\hline N'a bexo & Cinco pesos \\
\hline Küt'a bexo & Diez pesos \\
\hline R'et'a bexo & Cien pesos \\
\hline N'a nthebe bexo & Mil pesos \\
\hline N'a m'o bexo & Cien mil pesos \\
\hline N'a nthebe m'o bexo & Un millón de pesos \\
\hline N'a m'o ya m'o bexo &
\end{tabular}

Fuente: Propio autor.

\section{Los niños hñahñus y la escuela bilingüe}

Los niños y niñas asisten ${ }^{16}$ a una institución educativa conocida como "Escuela Primaria Bilingüe Indígena", en la que se maneja un curriculum oficial; las clases se dan en una sola lengua que es el español. La escuela en este contexto se convierte en un espacio donde

\footnotetext{
${ }^{16}$ Los alumnos y alumnas hñahñus pasan en la Escuela Primaria Bilingüe un promedio de 25 horas a la semana de lunes a viernes.
} 
interactúan sujetos que comparten sus conocimientos y a la vez construyen otros. Por ello radica la importancia de conocer la cultura de los sujetos, la red de significados que son propios de cada grupo y cómo cada uno de ellos puede ser beneficiado de esta interacción para construir y transformar la realidad que les toca vivir.

Los alumnos son portadores de saberes adquiridos en la familia, en la comunidad, pero que en el momento de entrar en contacto con la escuela, se encuentra con otro tipo de saberes que en la mayoría de los casos resulta ser contrastante. "La vida familiar y comunitaria tienen contrastes con las experiencias que los niños viven en la escuela, donde el aprendizaje se realiza de otra manera" (ROBLES, 1996: 13). Además de ser contenidos diferentes, también la dinámica de vida y de trabajo es totalmente diferente; porque pasan de una manera de aprender contextualizada haciendo, practicando, observando, a una forma de aprender escuchando, memorizando, analizando problemas descontextualizados y en muchos casos encerrados en las cuatro paredes.

En uno de los trabajos consultados, ALDAZ (1998) hace referencia a la importancia de sistematizar, organizar, escribir y tomar en cuenta los saberes de la comunidad del niño como punto de partida para que se inicie en la construcción de significados. Esta es una cuestión que necesariamente tiene que analizarse a través de varios estudios y posiblemente a través de proponer un cambio curricular.

Queda claro que la escuela es un espacio donde confluyen: la cultura escolar, entendida como el conjunto de conocimientos, contenidos, prácticas y opiniones pedagógicas de los maestros GIBAJA (1991) y la cultura del niño como aquellos conocimientos y saberes que ha adquirido en su familia y comunidad. Mismas que conforman la cultura instituida y la cultura instituyente (Cfr. CARRILLO, 1999: 17), donde se crean interacciones simbólicas con diferentes redes de significado.

Con relación a la condición social de cada familia, ésta se ve reflejada en el salón de clases, porque las familias de escasos recursos económicos hacen más uso de la lengua indígena, se observa que la cultura está muy arraigada en ellas y los niños presentan características específicas tales como: la forma de hablar, algunos se expresan menos; otros son más serios, callados, apáticos, porque temen a ser criticados o señalados por los errores que pueden cometer. 


\section{Conclusiones}

La idea de este artículo fue mostrar la compleja situación y la problemática que existe en la enseñanza de los contenidos matemáticos, la forma en que los alumnos aprenden y cómo expresan sus conocimientos en la resolución de problemas cotidianos en el medio indígena y obviamente esto no es retomado por la escuela bilingüe.

Se concluye que tanto en el contexto sociocultural comunitario como en el contexto escolar, existen procesos particulares de aprendizajes en niños que tienen características culturales y lingüísticas diferenciadas con sus propios ritmos y características; en este contexto, el aula se convierte en un espacio donde el conocimiento y los sujetos sociales se encuentran y que es precisamente en este lugar donde los problemas surgen, mismos que se manifiestan cuando los niños no pueden aplicar los conocimientos que son enseñados en la escuela.

Es sabido que los niños se apropian de la lengua indígena desde los primeros años de vida, también apreden a realizar actividades tales como ir a la leña, a ayudar en los quehaceres de la casa, en la siembra, en la albañilería, el pastoreo. etc. Los niños que asisten a la escuela bilingüe indígena, en su mayoría tienen un dominio del hñahñu y del español, aunque si hay muchos casos de niños que también dominan más la lengua indígena y caso de niños que dominan más el español. En este contexto se confirma el fenómeno de que el niño llega a la escuela con muy variados conocimientos que pueden servir como punto de partida para su aprendizaje de los contenidos escolares.

Cada niño es un portador de saberes adquiridos en la familia, en la comunidad, pero que en el momento de entrar en contacto con la escuela, se encuentra con otro tipo de saberes que en la mayoría de los casos resulta ser contrastante. "La vida familiar y comunitaria tienen contrastes con las experiencias que los niños viven en la escuela, donde el aprendizaje se realiza de otra manera" (ROBLES, 1996: 13). Además de ser contenidos diferentes, también la dinámica de vida y de trabajo es totalmente diferente; porque pasan de una manera de aprender contextualizada haciendo, practicando, observando; a una forma de aprender escuchando, memorizando, analizando problemas descontextualizados y en muchos casos encerrados en las cuatro paredes.

Resulta difícil entonces, para las niñas y niños indígenas, acceder al lenguaje matemático manejado por la cultura occidental, y cuando ellos llegan a entender algunos de los contenidos y manejar algunas habilidades, ya está terminando el ciclo escolar o un nivel educativo, cabe decir también que la continuidad educativa no es revisada y atendida por los maestros bilingües. 
Lo anterior significa que la enseñanza de las matemáticas también resulta ser un problema de comunicación, porque se abordan las matemáticas con un alto nivel de abstracción y de manera unilateral, caracterizada por la emisión y recepción de mensajes, esperando que siempre se produzcan cambios conductuales observables en los receptores. Ante esta situación resulta necesario crear las bases para una mejor comprensión de los contenidos escolares y habilidades matemáticas, a partir de los saberes etnomatemáticos y de la lengua que dominan las alumnas y alumnos indígenas.

Considero que conociendo la cultura del pueblo hñahñu, podemos entender su proceso de vida, sus formas de enseñar y aprender, así como las necesidades de aprendizaje que requieren para una formación más sólida que les permitan enfrentarse a los problemas de la vida cotidiana.

\section{Fuentes bibliográficas}

ALDAZ Hernández, Isaías (1992). Algunas actividades de los Mixes de Cacalotepec relacionadas con las matemáticas. Un acercamiento a su cultura. Tesis de Maestría. CINVESTAV/IPN. México.

(1998). Matemáticas y Etnomatemáticas. Cuadernillos para Maestros. Instituto Estatal de Educación Pública de Oaxaca (IEEPO). México.

BERNAL Pérez, Felipino (1996). Diccionario hñahñu-español, español-hñahñu, del Valle del Mezquital, Hidalgo. México, Talleres Gráficos de la Cámara de Diputados.

CASTRO Martínez, Encarnación et.al. (1989). "La acción en el aula y su planificación" en: Números y operaciones. Madrid: Síntesis.

CARRAHER, Terezinha, David W. Carraher y Analúcía D. Schliemann (1997a). "En la vida diez, en la escuela cero: los contextos culturales del aprendizaje de las matemáticas" en: Carraher, Terezinha, David W. Carraher y Analúcía D. Schliemann (1997) En la vida diez en la escuela cero. México, S. XXI Editores.

(1997b). "Matemáticas escritas versus matemáticas orales" en: Carraher, Terezinha, David W. Carraher y Analúcía D. Schliemann (1997). En la vida diez en la escuela cero. México, S. XXI Editores.

(1987). Tr. Eduardo Mancera "Matemáticas orales y escritas" tomado de Journal For Research in Mathematics Education. Vol. 18, No. 2, en:Revista Pedagogía. Matemáticas en el aula. Vol. 7 No. 21. 1997. México. UPN.

CARRILLO AVELAR, Antonio (1999). Los indígenas en la escuela bilingüe y su cultura instituida e instituyente. Tesis de Maestría. México. UAMI. 
D’ AMBROSIO, Ubiratán (1990). Etnomatemáticas. Atica, Serie fundamentos, Sao Paulo. Citado por Isaías Aldaz.

EZPELETA , J. Y E. Rockwell (1983). “Escuela y clases subalternas”. Cuadernos Políticos 37. México. Era.

FUENLABRADA, Irma (1997). La didáctica, los maestros y el conocimiento matemático. México, Documento DIE 43, CINVESTAV/IPN.

GIBAJA, Regina E. (1991). La cultura de la escuela. Creencias pedagógicas y estilos de enseñanza. Buenos Aires. AIQUE.

GÓMEZ Muñoz, Margarita (1997). "Los aprendizajes comunitarios en los altos de Chiapas”. Pp. 55-84. En: María bertely Busquets y adriana Robles Valle.(1997). Indígenas en la escuela. México. Investigación Educativa 1993-1995.

MARTín C. Donaciana, Victorino V. Gómez B. y Pedro M. Godínez S. (1986). Ra Thogui n’e yä nfädi yä ñähñu. La historia y los conocimientos de los ñähñu. México. DGEI. Centro Social de Gardonal.

ROBLES Valle, Adriana (1996). Diálogo cultural: Tiempo mazahua en un jardín de niños rural. México, Tesis/DIE/CONACYT.

SEP (1996). Monografía de Hidalgo. Educación Básica Primaria. México,

VÁZQUEZ Valdivia, Héctor. (1995) “Los otomíes del Valle del Mezquital” en: Etnografía Contemporánea de los Pueblos Indígenas de México. Región Centro. México. INI/SEDESOL.

VILLAVICENCIO Ubillús, Martha (1987). Ponencia presentada en la VII Conferencia Intreramericana de Educación Matemática. Santo Domingo, Julio/87. (1990). La matemática en la educación bilingüe: el caso de Puno. Perú.

ZALAVSKY s/n, (1989). En International study group on ethnomathematics (ISGEM). USA. Newsletter, vol. 5, No. 1, diciembre.

Submetido em 04 de julho de 2019.

Aceito em 16 de janeiro de 2020.

Publicado em 21 de janeiro de 2020. 